psychopraxis. neuropraxis $2021 \cdot 24: 310-315$ https://doi.org/10.1007/s00739-021-00746-9 Angenommen: 2. August 2021

Online publiziert: 31. August 2021

(c) Der/die Autor(en) 2021

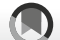

Check for
updates

\section{Dagmar Steinmair ${ }^{1,2} \cdot$ Felicitas Datz $^{1} \cdot$ Henriette Löffler-Stastka ${ }^{1}$}

'Klinik für Psychoanalyse und Psychotherapie, Medizinische Universität Wien, Wien, Österreich

${ }^{2}$ Karl Landsteiner Privatuniversität für Gesundheitswissenschaften, Krems an der Donau, Österreich

\title{
Klinische Erfahrung und deren Auswirkungen auf Interventionstechnik und Working Alliance
}

\section{Einleitung}

Erklärungsmodelle für „clinical reasoning“, welche sich aus dem Kontext klinischen Arbeitens entwickeln, orientieren sich häufig an Nützlichkeit und Effizienz. Die Suche nach neuen Hypothesen und Fragestellungen, wenn signifikante klinische psychische Phänomene nicht mit gängigen Theorien und Lösungswegen zu erklären und behandeln sind, kann der Beginn einer Forschungsidee auf dem Weg zu Innovation sein [1]. Wissenschaftliche Erkenntnisse aus der Psychotherapieforschung generieren eine Vielfalt an Erklärungsansätzen und Theorien, welche sich in "Akkuratesse, Reichweite, Einfachheit und Fruchtbarkeit" unterscheiden [2]. Mit der Dauer der klinischen Erfahrung nimmt in Therapeuten tendenziell die Bereitschaft $\mathrm{ab}$, neue evidenzbasierte wissenschaftliche Erkenntnisse in ihre Tätigkeit einfließen zu lassen [3]. Ein intuitiver, individueller Behandlungsstil entwickelt sich, ausgehend von einer effektiveren und besseren klinischen Entscheidungsfähigkeit.

\section{》) Interventionstechniken verändern sich basierend auf intuitiven Prozessen}

Die therapeutische Beziehung („allian$\mathrm{ce}^{\text {") }}$ entspricht nach moderner Konzeption einer „authentischen“ menschlichen Beziehung und stellt den Kern der Psychotherapie und psychotherapeutischen
Medizin dar [4, 5]. Die bisherige Evidenz weist darauf hin, dass klinische Erfahrung und berufliches Training nicht direkt zu einer Verbesserung der Arzt/ Therapeut-Patient Beziehung („working alliance") beitragen [6-9]. Interpersonelle Fähigkeiten korrelierten jedoch unabhängig von der Erfahrung mit besseren therapeutischen Beziehungen und erfolgreichen Therapieergebnissen. Allerdings erkennen erfahrene Ärzte/ Therapeuten die Wichtigkeit der therapeutischen Beziehung eher an und setzen gezielt einen Fokus darauf [10]. Zudem verändern sich Interventionstechniken basierend auf intuitiven Prozessen, welche sich wiederum mit der Erfahrung verändern [11]. Als wichtige veränderliche intuitive Prozesse in Ärzten/ Therapeuten werden bewusste (z. B. die Anwendung von Regeln, Reflexion) von unbewussten (emotionales Arousal, Suche nach Alternativen, Automatisierung) unterschieden [11]. Interventionstechniken haben direkten Einfluss auf die therapeutische Beziehung (z.B. bedeutungsgebende Interpretationen und Deutungen basierend auf Gegenübertragung und Übertragung, vgl. [12])

Nonverbale, affektive Aspekte der therapeutischen Interaktion sind von zentraler Bedeutung für die Untersuchung ärztlich-therapeutischer Wirksamkeiten [12]. Die Fähigkeit der Reverie, (träumerisches Ahnungsvermögen nach Bion), ermöglicht Ärzten/Therapeuten unter anderem Containment. Projektionen, also unerträgliche Gefühle und innere Zustände des Patienten, können bei ad- äquatem Containment zuerst im Arzt/ Therapeuten aufgenommen, dann verarbeitet und in etwas Erträgliches oder Bedeutungsvolles modifiziert, vom Patienten wieder aufgenommen werden (Introjektion) [13-15].

\section{Beziehungsdynamik und „interaction structure}

Psychotherapie, so könnte man sagen, bedeutet Beziehung und löst im Arzt/ Therapeuten unweigerlich emotionale Reaktionen aus. Die Informationen, welche aus diesen Reaktionen potenziell über den Patienten gewonnen werden können, zu nutzen, ist essenzieller Bestandteil psychotherapeutischer Techniken [16]. Holmquist spricht in diesem Zusammenhang vom emotionalen Universum des Arztes/Therapeuten, welches entscheidender zur Gegenübertragungsoder emotionalen Reaktion beiträgt als Einflüsse von Patienten allein. In diesem individuellen Erfahrungsraum finden sich für jeden Arzt/Therapeuten spezifische und unspezifische affektive Reaktionen.

Kontrovers wird jedoch diskutiert, welche Implikationen sich für die Therapie aus individuellen Interaktions- und Reaktionsmustern sowie aus Abweichungen davon ergeben und inwieweit sich diese über die Zeit verändern und modifizieren lassen (z.B. Selbsterfahrung, Supervision, Lehrtherapie). Ein Zusammenhang zwischen der Persönlichkeit von Patienten und der emotionalen Reaktion von Therapeuten war in einer 
Untersuchung von Colli et al. (2014) [17] deutlicher als ein Zusammenhang mit anderen psychischen Begleitdiagnosen (17, N = 149, Patientinnen mit Essstörungen), unabhängig vom üblichen Erleben der Therapeuten. Holmquists klinische Untersuchung stützte die Hypothese, dass sich für Therapeuten Muster zeigen, wie sie auf „Patienten im Allgemeinen“ emotional reagieren. Weicht das Empfinden eines Therapeuten also von seinem üblichen Muster $a b$, indem sich in der Interaktion mit einem bestimmten Patienten oder in individuellen Therapiesitzungen Abweichungen zeigen, ist dies bemerkenswert. Die entsprechenden Ergebnisse waren für verschiedene Therapeuten ähnlich nachweisbar [16]. Freilich muss diese Hypothese vorsichtig diskutiert werden; interpersonelle Fähigkeiten von Therapeuten sind zwar mit als einer der Parameter für Therapieerfolge diskutiert worden, allerdings lässt sich der Einfluss von Psychotherapeuten als Person schwer operationalisieren [18]. Emotionen sind Dimensionen inneren Erlebens, damit ist die Beschreibung individuell (Erleben, Ausdruck, körperlicher Zustand). Finden sich im Arzt/ Therapeuten allerdings durchgängig hohe Werte für „das Gefühl, abgeschottet zu werden“, hat dies einen negativen Einfluss auf den Therapieverlauf, besonders bei Patienten mit schlechten Bindungserfahrungen [19]. Einflüsse von Patienten zeigten sich in Holmquists Untersuchung vor allem darin, wie sehr Therapeuten sich „hilfreich“ oder „frustriert“ fühlten. Gaben Therapeuten an, sich gelangweilt, ängstlich, manipuliert, infrage gestellt, ausgeschlossen oder misstrauisch zu erleben, hatte dies eher mit den jeweiligen Patienten und Therapieverläufen zu tun als mit Therapeutencharakteristika. Ob sich Therapeuten jedoch überwältigt oder müde fühlten, war über den Beobachtungszeitraum der Holmquist-Studie innerhalb derselben Therapie stabil [16].

\section{》) Das Eingehen auf negative Spitzenaffekte trägt zum Therapieerfolg bei}

Lassen sich also Generalisierungen treffen, welche Reaktionen in gewissen Situa- tionen erwartbar sind, und welche weniger? Diese Frage ist viel diskutiert die bisherige Evidenz weist darauf hin, dass Verallgemeinerungen über „normale emotionale Reaktionen im Therapeuten" sehr schwierig sind. Supervision sollte $\mathrm{zu}$ einem besseren Verständnis der Gefühle und Einstellungen in Ärzten/ Therapeuten führen.

Jede Arzt/Therapeut-Patient-Dyade muss in ihrem Kontext betrachtet werden; der Begriff des „emotionalen Universums“ des Therapeuten trifft wohl sehr gut die Beschreibung eines über die Zeit und klinische Erfahrung stabilen individuellen Bezugsrahmens, welcher durch neue zwischenmenschliche Interaktionen stetig erweitert, aber im Wesentlichen nicht erschüttert wird. Studien zur therapeutischen Interaktion lassen unabhängig davon Schlüsse über psychopathologiespezifisches, mimischaffektives Verhalten und über typische Interaktionsmuster und Beziehungsgestaltung zu [20].

Die Entstehung einer positiven Arzt/ Therapeut-Patient-Interaktion („working alliance") ist entscheidend für den Therapieerfolg $[21,22]$. Für die Behandlung von depressiven Patienten zeigten sich in der Studie von Datz et al. bei erfahrenen Therapeuten spezifische Interaktionsmuster während therapeutischer Sitzungen, welche sich im Therapieverlauf änderten [22, 23]. Die herausgearbeitete Interaktionsstruktur basiert auf den Reaktionen und Gefühlen von Therapeuten in 639 Sitzungen (vgl. Münchner Psychotherapiestudie: 639 Therapiesitzungen von 100 Patienten, 21 erfahrene Therapeuten; [23, 24]). Bei erfahrenen Therapeuten waren innere (emotionale) Reaktionsweisen und äußere Reaktionsweisen (Therapeutenverhalten) unabhängige Faktoren im Vergleich zu ihrer emotionalen Befindlichkeit („feindselige Gefühle“, „positive Gefühle“, „Gefühl, abgeschottet zu werden“, „überwältigt“, „Gefühl, überinvolviert zu sein") [25]. Entscheidende Faktoren für das Zustandekommen einer stabilen Arzt/Therapeut-PatientBeziehung mit gutem Therapieergebnis war ein konstruktiver Umgang mit der Übertragung und Gegenübertragungsreaktion. $\mathrm{Zu}$ Beginn der Therapie waren häufig feindselige Gefühle und eine gewisse Distanziertheit und Uneinigkeit von Patientenseite angegeben worden. Im weiteren Therapieverlauf entwickelte sich jedoch aus einem initial nicht übereinstimmenden Interaktionsmuster eine positive/befriedigende Gegenübertragung. Die emotionale Interaktion zwischen dem Arzt/Therapeuten und Patient geht, wenn die positive Übertragung mit einer positiven Gegenübertragung konkordant beantwortet wird, mit der Etablierung eines positiven Introjekts einher [26].

In einer weiteren Untersuchung [27] wurde der Fokus auf das mimisch-affektive Interaktionsmuster gelegt. Mikroexpressionen können mittels Videoanalyse und Facial-Action-Coding-System erkannt und klassifiziert werden [28]. Es zeigte sich, dass das Eingehen auf negative Spitzenaffekte, wie bspw. Ekel oder Verachtung, welche sich in der Mimik gegenseitig ausdrücken, zum Therapieerfolg beiträgt. Dies trifft zu, wenn diese Interaktionen adäquat durchgearbeitet werden. Konfrontation und interpretative Interventionen tragen eher zur Bedeutungsgebung bei als supportive Interventionen. Beschränken sich die therapeutischen Maßnahmen auf ebendiese Interventionen, entwickeln sich weniger tragfähige therapeutische Beziehungen. Die Bedeutsamkeit der Untersuchung therapeutischer Mikroprozesse und insbesondere affektiv-kognitiver Aspekte in der Interaktion ist evident.

\section{Kasuistik}

„... ich bin wieder bei Kräften, sag ich mal“, er werde das Studium nun doch nicht unterbrechen, sagt N. Aufgrund einer mittelgradigen depressiven Episode ist Herr N (20-jährig, Student) zum ersten Mal hospitalisiert, der Aufenthalt geht dem Ende zu. In Stresssituationen hatte N auch in der Vergangenheit immer wieder depressiv reagiert, mit vollständiger Remission der Symptomatik auch ohne Behandlung. Die Leistungsansprüche von Herrn $\mathrm{N}$ an sich selbst sind hoch. Er zeigt sich im Gespräch konzentriert und ruhig, im Tempo adäquat, seine Gedankengänge sind kohärent, ausgeglichen. Herr N denkt zurück an den Beginn dieser Epi- 
sode (schüttelt den Kopf, zeigt für einige Millisekunden Verachtung in den microexpressions) „wie ich da am Sonntag kommen bin, da habeich gedacht, ich verbring da a ganzes Monat [...]“. Das Gesicht von $\mathrm{N}$ hellt sich zusehends auf. Die gedrückte Stimmung, das Gefühl der Überforderung und das Erleben des Patienten, nicht mehr belastbar zu sein, haben sich bereits gebessert. Der behandelnde Psychiater setzt den Fokus dann auf das Jetzt, er strahlt Interesse (zeigt für einige Millisekunden positive Affekte in den micro-expressions) und Wohlwollen aus. Herr N wirkt noch affektarm, jedoch adäquater affizierbar. Es ist sichtbar, dass ein Vertrauensverhältnis besteht. „Das alles einmal rauslassen, über alles einmal sprechen" habe ihm am meisten geholfen. Durch Nachfragen regt der Psychiater $\mathrm{N}$ dazu an, darüber zu reflektieren, wie es nun weitergeht. Es wird klar, dass noch einige organisatorische Unklarheiten bestehen. Es folgt eine sorgfältige Aufklärung und Besprechung des weiteren Behandlungsplans, wobei der Psychiater auf Transparenz achtet. Das verordnete Antidepressivum wirkt bei Herrn $\mathrm{N}$ eigentlich zu früh, darauf geht der Psychiater ein „... es kann so eine Art Bereitschaft sein, dann spricht man generell auf Medikamente gut an." (Placebo-Effekt). Herr $\mathrm{N}$ wird die Medikamente für das Wochenende noch mitbekommen, für die Zeit unmittelbar danach erhält er ein Rezept. Anschließend ist die weitere therapeutische Behandlung im ambulanten Setting Thema. Aufgrund des rezidivierenden Verlaufs wird eine Dauertherapie für 1-2 Jahre mit Sertralin notwendig; Herr $\mathrm{N}$ nickt - er hat die Implikationen der Diagnose akzeptiert und verfolgt aufmerksam die Empfehlungen für die weitere Therapie. Sowohl eine psychotherapeutische als auch eine psychiatrische Behandlung wird noch während des stationären Aufenthaltes in die Wege geleitet. Vgl. auch [27].

\section{Diskussion und Ausblick}

In der geschilderten Therapiesequenz, welche mittels Videoanalyse untersucht wurde, zeigte der Arzt/Therapeut initial komplementäre Affektmuster und gegen Ende hin reziproke. Die zu frühe

psychopraxis. neuropraxis $2021 \cdot 24: 310-315$ https://doi.org/10.1007/s00739-021-00746-9

c) Der/die Autor(en) 2021

\section{Steinmair · F. Datz · H. Löffler-Stastka \\ Klinische Erfahrung und deren Auswirkungen auf Interventionstechnik und Working Alliance}

\section{Zusammenfassung}

Die Wichtigkeit einer tragenden

Arzt/Therapeut-Patient-Beziehung

konnte als der wesentliche Faktor für den

Therapieerfolg herausgearbeitet werden. Mit zunehmender klinischer Erfahrung verbessert sich die Fähigkeit von Behandlern, auch in komplexeren klinischen Situationen akkurate diagnostische und therapeutische Entscheidungen zu treffen. Bisher konnte zwar kein direkter Einfluss der klinischen Erfahrung auf die therapeutische Allianz nachgewiesen werden, allerdings wird deutlich, dass sich Interventionstechnik und damit Interaktionen bei erfahrenen Ärzten/Therapeuten basierend auf intuitiven Prozessen verändern. Konstruktive Interaktionsprozesse bilden das Fundament einer stabilen Arzt/TherapeutPatient-Beziehung. Analysen nonverbaler Interaktionsaspekte zeigten, dass sich die Bearbeitung sogenannter negativer Spitzenaffekte in der Therapie günstig auf die therapeutische Beziehung und auf den Therapieerfolg auswirken.

Schlüsselwörter

Interaktionsstruktur - Gegenübertragung . Therapeutische Beziehung · Affektive Präsenz . Verarbeitungsprozesse

\section{Clinical experience and its effects on intervention technique and working alliance}

\section{Abstract}

The importance of a a supportive doctor/therapist-patient relationship has been identified as an essential factor for therapy success. With increasing clinical experience, the ability of practitioners to make accurate diagnostic and therapeutic decisions improves, even in more complex clinical situations. Although no direct influence of clinical experience on the therapeutic alliance could be demonstrated so far, it is clear that the intervention technique and thus interactions change in experienced doctors/therapists based on intuitive processes. Constructive interaction processes build the foundations of a stable doctor/therapist-patient relationship. Analyses of nonverbal aspects of interaction show that working through negative affects has a favourable effect on the therapeutic relationship and on therapy success.

\section{Keywords}

Interaction structure - Countertransference . Therapeutic relationship - Emotional arousal Containment
Wirkung des Psychopharmakons kann im übertragenen Sinne für positive Introjektionen stehen, welche durch die positiven Erfahrungen im Rahmen des stationären Aufenthaltes gemacht wurden - bei anfänglicher Distanziertheit, Rückzug und Verzweiflung im Patienten. Die initiale Nichtübereinstimmung in mimischen Mustern und emotionaler Reaktion kann für frühe (externalisierende) Abwehrreaktionen in der Phase des Beziehungsaufbaus sprechen. Diese Betrachtung dieser einzelnen Therapiesitzung passt zu den Ergebnissen der in der Einleitung dargestellten Untersuchungen zur Interaktionsstruktur über ganze Therapieverläufe [23].
Gegenübertragungsreaktionen in Therapeuten sind in der Literatur als meistens vom Therapeuten wahrgenommene Phänomene beschrieben (vgl. [29]: $80 \%$ der Sitzungen), allerdings waren sie innerhalb dieser Sitzungen als nicht häufiges Phänomen beschrieben. Viele Untersuchungen verließen sich hierbei auf Selbstevaluierungen der Therapeuten, objektive Messungen sind schwieriger, lassen aber häufigere Gegenübertragungsphänomene vermuten als subjektiv erwähnt.

Zusammenfassend lässt sich sagen, dass klinische Erfahrung sich nicht automatisch auf die Güte der therapeutischen Beziehung auswirken wird. Interpersonelle Fähigkeiten sind dem 
Hier steht eine Anzeige.

\section{曾 Springer}


Arzt/Therapeuten inhärent, die therapeutische Beziehung ist wichtig für eine erfolgreiche Therapie. Die Erforschung der Faktoren, welche zur Etablierung einer guten „working alliance“ beitragen, kann zeigen, wie diese Beziehung verbessert werden kann. Das Durcharbeiten negativer Spitzenaffekte und die Kenntnis von üblichen Interaktionsmustern in Therapieverläufen sind Ansatzpunkte.

\section{Fazit für die Praxis \\ - Klinische Erfahrung alleine trägt nicht automatisch zur Verbesserung der therapeutischen Beziehung bei. \\ - Emotionales Erleben von Thera- peuten ist entscheidend für den Therapieerfolg. Insbesondere der Umgang mit der Übertragung und Gegenübertragung wirkt sich auf den Therapieerfolg aus. \\ - Interaktionsstrukturen und Muster zeigen einen typischen Verlauf in einer erfolgreichen Therapie, dies zeigte sich unabhängig von der ange- wandten therapeutischen Methode und innerhalb einer therapeutischen Sitzung wie auch für den Therapie- verlauf. \\ - Die adäquate Bearbeitung von ne- gativen Spitzenaffekten trägt zur Verbesserung der Arzt/Therapeut- Patient-Beziehung und zum Thera- pieerfolg bei.}

\section{Korrespondenzadresse}

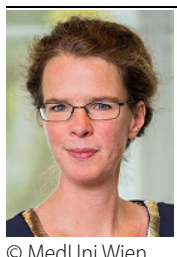

Univ. Prof. Dr. med. univ. Henriette Löffler-Stastka Klinik für Psychoanalyse und Psychotherapie, Medizinische Universität Wien Währinger Gürtel 18-20, 1090 Wien, Österreich henriette.loefflerstastka@meduniwien.ac.at

Funding. Open access funding provided by Medical University of Vienna.

\section{Einhaltung ethischer Richtlinien}

Interessenkonflikt. D. Steinmair, F. Datz und H. Löffler-Stastka geben an, dass kein Interessenkonflikt besteht.
Für diesen Beitrag wurden von den Autoren keine Studien an Menschen oder Tieren durchgeführt. Für die aufgeführten Studien gelten die jeweils dort angegebenen ethischen Richtlinien.

Open Access. Dieser Artikel wird unter der Creative Commons Namensnennung 4.0 International Lizenz veröffentlicht, welche die Nutzung, Vervielfältigung Bearbeitung, Verbreitung und Wiedergabe in jeglichem Medium und Format erlaubt, sofern Sie den/die ursprünglichen Autor(en) und die Quelle ordnungsgemäß nennen, einen Link zur Creative Commons Lizenz beifügen und angeben, ob Änderungen vorgenommen wurden.

Die in diesem Artikel enthaltenen Bilder und sonstige Drittmaterial unterliegen ebenfalls der genannten Creative Commons Lizenz, sofern sich aus der Abbildungslegende nichts anderes ergibt. Sofern das betreffende Material nicht unter der genannten Creative Commons Lizenz steht und die betreffende Handlung nicht nach gesetzlichen Vorschriften erlaubt ist, ist für die oben aufgeführten Weiterverwendungen des Materials die Einwilligung des jeweiligen Rechteinhabers einzuholen.

Weitere Details zur Lizenz entnehmen Sie bitte de izenzinformation auf http://creativecommons.org/ licenses/by/4.0/deed.de.

\section{Literatur}

1. Govrin A (2019) Facts and sensibilities: What is a psychoanalytic innovation? Front Psychol 10:1781. https://doi.org/10.3389/fpsyg.2019. 0178

2. Kuhn TS (1970) Reflections on my critics. In: Lakatos I, Musgrave A (Hrsg) Criticism and growth of knowledge.Cambridge University Press, Cambridge, S231-278

3. Dawson GC (2018) Years of clinical experience and therapist professional development: a literature review. J Contemp Psychother 48:89-97. https:// doi.org/10.1007/s10879-017-9373-8

4. Parth K, Datz F, Seidman C, Löffler-Stastka H (2017) Transference and countertransference: a review. Bull Menninger Clin 81(2):167-211. https://doi. org/10.1521/bumc.2017.81.2.167

5. Safran JD, Muran JC (2006) Has the concept of the therapeutic alliance outlived its usefulness? Psychotherapy 43(3):286-291. https://doi.org/10. 1037/0033-3204.43.3.286

6. Dunkle JH, Friedlander ML (1996) Contribution of therapist experience and personal characteristics to the working alliance. J Couns Psychol 43:456-460. https://doi.org/10.1037/0022-0167. 43.4 .456

7. Tracey TJ, Kokotovic AM (1989) Factor structure of the working alliance inventory. Psychol Assess 1:207-210

8. Hersoug AG, Hoglend P, Monsen J, Havik OE (2001) Quality of working alliance in psychotherapy: therapist variable and patient/therapist similarity as predictors. JPsychother Pract Res 10:205-216

9. Gelso CJ, Kelley FA, Fuertes JN, Marmarosh C Holmes SE, Costa C et al (2005) Measuring the real relationship in psychotherapy: initial validation of the therapist form. J Couns Psychol 52:640-649. https://doi.org/10.1037/0022-0167.52.4.640

10. Rønnestad MH, Skovholt TM (2003) The journey of the counselor and therapist: research findings and perspectives on professional development. JCareer Dev 30:5-44
11. CasparF(1997)What goes on in a psychotherapist's mind? Psychother Res 7(2):105-125. https://doi. org/10.1080/10503309712331331913

12. Löffler-Stastka H, Datz F, Parth K, Preusche I, Bukowski X, Seidman C (2017) Empathy in psychoanalysis and medical education-What can we learn from each other? BMCMed Educ 17(1):74 https://doi.org/10.1186/s12909-017-0907-2

13. Bion W (1997) Lernen durch Erfahrung. Suhrkamp, Frankfurt/Main

14. Lacan J (1966) Das Spiegelstadium als Bildner der Ichfunktionen [1949]. In: von Haas N (Hrsg) Lacan: Schriften, Bd. 1. Walter, Olten, S61-70

15. Winnicott DW (1979) Die Spiegelfunktion von Mutter und Familie in der kindlichen Entwicklung. In: Winnicott DW (Hrsg) Vom Spiel zur Kreativität. Klett-Cotta, Stuttgart, S128-135

16. Holmqvist R (2001) Patterns of consistency and deviation in therapists' countertransference feelings. JPsychother Pract Res 10(2):104-116

17. Colli A, Tanzilli A, Dimaggio G, Lingiardi V (2014) Patient personality and therapist response: an empirical investigation. Am J Psychiatry 171(1):102-108. https://doi.org/10.1176/appi. ajp.2013.13020224

18. Sonnenmoser M (2014) Einfluss des Psychotherapeuten auf den Therapieerfolg: Gut untersucht und doch unvorhersagbar. Dtsch Arztebl 13:312

19. Dahl HJ, Høglend P, Ulberg R, Amlo S, Gabbard GO, Perry JC, Christoph PC (2017) Does therapists' disengaged feelings influence the effect of transference work? A study on countertransference. Clin Psychol Psychother 24(2):462-474. https:// doi.org/10.1002/cpp.2015

20. Arboleda L, Zschokke V (2014) Die Borderlinestörung gesprächs- und erzählanalytisch betrachtet. Psychosozial-Verlag, Giessen

21. Falkenström F, Ekeblad A, Holmqvist R (2016) Improvement of the working alliance in one treatment session predicts improvement of depressive symptoms by the next session. J Consult Clin Psychol 84(8):738-751. https://doi. org/10.1037/ccp0000119

22. Datz F, Parth K, Said U, Löffler-Stastka H (2016) Countertransference triggered activity in treatment. Good, bad or useful? Br J Med Med Res 11(9):1-11. https://doi.org/10.9734/BJMMR/ 2016/21985

23. Datz F, Parth K, Rohm C, Madanoglu S, Seidman C, Löffler-Stastka H (2016) Dimensions of activity in countertransference and therapist reactions: therapist reactions during sessions with depressed patients. Z Psychosom Med Psychothe 62(4):322-335. https://doi.org/10.13109/zptm. 2016.62.4.322

24. Huber D, Zimmermann J, Henrich G, Guenther K (2014) Comparison of cognitive-behaviour therapy with psychoanalytic and psychodynamic therapy for depressed patients - a three year follow-up study. Z Psychosom Med Psychother 58(3):299-316. https://doi.org/10.13109/zptm. 2012.58.3.299

25. Löffler-Stastka H, Grassl R (2006) Fragebogen zur Gegenübertragung - Manual. Medizinische Universität Wien, Wien (dt. Übersetzung zu Drew Westen (2005) Countertransference questionnaire - (TQ)

26. Zimmermann J, Löffler-Stastka H, Huber D, Klug G, Alhabbo S, Bock A, Benecke C (2015) Is it all about the higher dose? Why psychoanalytic therapy is an effective treatment for major depression. Clin Psychol Psychother 22(6):469-487. https://doi. org/10.1002/cpp.1917 
27. Datz F, Wong G, Löffler-Stastka H (2019) Interpretation and working through contemptuous facial micro-expressions benefits the patienttherapist relationship. Int J Environ Res Public Health 16(24):4901. https://doi.org/10.3390/ ijerph16244901

28. Ekman P, Friesen WV, Ancoli S (1980) Facial signs of emotional experience. J Pers Soc Psychol 39(6):1125-1134. https://doi.org/10. 1037/h0077722

29. Hayes JA, McCracken JE, Hill CE, McClanahan MK, Harp JS, Carozzoni P (1998) Therapist perspectives on countertransference: qualitative data in search of a theory.J Couns Psychol 45(4):468-482

30. Gelso CJ, Hayes JA (2007) Countertransference and the therapist's inner experience. Perils and possibilities. Lawrence Erlbaum, New Jersey

Hinweis des Verlags. Der Verlag bleibt in Hinblick auf geografische Zuordnungen und Gebietsbezeichnungen in veröffentlichten Karten und Institutsadressen neutral.
Hier steht eine Anzeige.

Springer 